\title{
ASYMPTOTIC PREDICTIVE INFERENCE WITH EXCHANGEABLE DATA
}

\author{
PATRIZIA BERTI, LUCA PRATELLI, AND PIETRO RIGO
}

\begin{abstract}
Let $\left(X_{n}\right)$ be a sequence of random variables, adapted to a filtration $\left(\mathcal{G}_{n}\right)$, and let $\mu_{n}=(1 / n) \sum_{i=1}^{n} \delta_{X_{i}}$ and $a_{n}(\cdot)=P\left(X_{n+1} \in \cdot \mid \mathcal{G}_{n}\right)$ be the empirical and the predictive measures. We focus on$$
\left\|\mu_{n}-a_{n}\right\|=\sup _{B \in \mathcal{D}}\left|\mu_{n}(B)-a_{n}(B)\right|
$$

where $\mathcal{D}$ is a class of measurable sets. Conditions for $\left\|\mu_{n}-a_{n}\right\| \rightarrow 0$, almost surely or in probability, are given. Also, to determine the rate of convergence, the asymptotic behavior of $r_{n}\left\|\mu_{n}-a_{n}\right\|$ is investigated for suitable constants $r_{n}$. Special attention is paid to $r_{n}=\sqrt{n}$ and $r_{n}=\sqrt{\frac{n}{\log \log n}}$. The sequence $\left(X_{n}\right)$ is exchangeable or, more generally, conditionally identically distributed.
\end{abstract}

\section{INTRODUCTION}

Throughout, $S$ is a Borel subset of a Polish space and

$$
X=\left(X_{n}: n \geq 1\right)
$$

a sequence of $S$-valued random variables on a probability space $(\Omega, \mathcal{A}, P)$. Further, $\mathcal{G}=\left(\mathcal{G}_{n}: n \geq 0\right)$ is a filtration on $(\Omega, \mathcal{A}, P)$ and $\mathcal{B}$ is the Borel $\sigma$-field on $S$ (thus, $\mathcal{B}$ is generated by the relative topology that $S$ inherits as a subset of a Polish space). We fix a subclass $\mathcal{D} \subset \mathcal{B}$ and we let $\|\cdot\|$ denote the sup-norm over $\mathcal{D}$, namely

$$
\|\alpha-\beta\|=\sup _{B \in \mathcal{D}}|\alpha(B)-\beta(B)|
$$

whenever $\alpha$ and $\beta$ are probability measures on $\mathcal{B}$.

Let

$$
\mu_{n}=(1 / n) \sum_{i=1}^{n} \delta_{X_{i}} \quad \text { and } \quad a_{n}(\cdot)=P\left(X_{n+1} \in \cdot \mid \mathcal{G}_{n}\right) .
$$

Both $\mu_{n}$ and $a_{n}$ are random probability measures on $\mathcal{B} ; \mu_{n}$ is the empirical measure and (if $X$ is $\mathcal{G}$-adapted) $a_{n}$ is the predictive measure.

Under some conditions, $\mu_{n}(B)-a_{n}(B) \stackrel{a . s}{\longrightarrow} 0$ for fixed $B \in \mathcal{B}$. In that case, a question is whether $\mathcal{D}$ is such that $\left\|\mu_{n}-a_{n}\right\| \stackrel{a . s}{\longrightarrow} 0$. As discussed in Section 2, such a question naturally arises in several frameworks, including Bayesian consistency and frequentistic approximation of Bayesian procedures.

In this paper, conditions for $\left\|\mu_{n}-a_{n}\right\| \longrightarrow 0$, almost surely or in probability, are given. Also, to determine the rate of convergence, the limit behavior of $r_{n}\left\|\mu_{n}-a_{n}\right\|$ is investigated for suitable constants $r_{n}$. Special attention is paid to $r_{n}=\sqrt{n}$ and

2010 Mathematics Subject Classification. 60G09, 60B10, 60A10, 60G57, 62F15.

Key words and phrases. Bayesian consistency - Conditional identity in distribution - Empirical measure - Exchangeability - Predictive measure - Random probability measure. 
$r_{n}=\sqrt{\frac{n}{\log \log n}}$. Various new results are proved. In addition, to get a reasonably complete picture, a few known facts from [2]-[5] are connected and unified.

The sequence $X$ is assumed to be exchangeable or, more generally, conditionally identically distributed. We refer to Section 3 for conditionally identically distributed sequences, and we recall that $X$ is exchangeable if $\left(X_{j_{1}}, \ldots, X_{j_{n}}\right) \sim$ $\left(X_{1}, \ldots, X_{n}\right)$ for all $n \geq 1$ and all permutations $\left(j_{1}, \ldots, j_{n}\right)$ of $(1, \ldots, n)$.

We next briefly state some results. We assume a mild measurability condition on $\mathcal{D}$, called countable determinacy and introduced in Section 3. For the sake of simplicity, we take $X$ exchangeable and $\mathcal{G}=\mathcal{G}^{X}$, where

$$
\mathcal{G}_{0}^{X}=\{\emptyset, \Omega\} \quad \text { and } \quad \mathcal{G}_{n}^{X}=\sigma\left(X_{1}, \ldots, X_{n}\right), \quad n \geq 1,
$$

is the filtration induced by $X$. We also recall that, since $X$ is exchangeable, there is a (a.s. unique) random probability measure $\mu$ on $\mathcal{B}$ such that $\mu_{n}(B) \stackrel{a . s}{\longrightarrow} \mu(B)$ for each $B \in \mathcal{B}$; see e.g. [1].

Then, $\left\|\mu_{n}-a_{n}\right\| \stackrel{\text { a.s. }}{\longrightarrow} 0$ with $\mathcal{D}=\mathcal{B}$ provided $\mu$ is a.s. discrete; see Example 4. This simple fact may be useful in Bayesian nonparametrics, for $\mu$ is a.s. discrete under most popular priors. Indeed, examples of nonparametric priors which lead to a discrete $\mu$ are: Dirichlet [26], two-parameter Poisson-Dirichlet [24], normalized completely random measures [20], Gibbs-type priors [12] and beta-stacy [23].

Another useful fact (Theorem 2 and Corollary 3 ) is that

$$
\limsup _{n} \sqrt{\frac{n}{\log \log n}}\left\|\mu_{n}-a_{n}\right\| \leq \sqrt{2 \sup _{B \in \mathcal{D}} \mu(B)(1-\mu(B))} \text { a.s. }
$$

provided $\mathcal{D}$ is a VC-class. Unlike the i.i.d. case, inequality (1) is not sharp. If $X$ is exchangeable, it may be even that $n\left\|\mu_{n}-a_{n}\right\|$ converges a.s. to a finite limit. This happens, for instance, when the probability distribution of $X$ is of the FergusonDirichlet type, as defined in Subsection 4.2; see also forthcoming Theorem 6. Even if not sharp, however, inequality (1) provides a meaningful information on the rate of convergence of $\left\|\mu_{n}-a_{n}\right\|$ when $X$ is exchangeable and $\mathcal{D}$ a VC-class.

The notion of VC-class is recalled in Subsection 4.1 (before Corollary 3). VCclasses are quite popular in frameworks such as empirical processes and statistical learning, and in real problems $\mathcal{D}$ is often a VC-class. If $S=\mathbb{R}^{k}$, for instance, $\mathcal{D}=\left\{\left(-\infty, t_{1}\right] \times \ldots \times\left(-\infty, t_{k}\right]:\left(t_{1}, \ldots, t_{k}\right) \in \mathbb{R}^{k}\right\}, \mathcal{D}=\{$ half spaces $\}$ and $\mathcal{D}=\{$ closed balls $\}$ are VC-classes.

A further result (Corollary 8) concerns $r_{n}=\sqrt{n}$. Let

$$
a_{n}^{*}(B)=P\left\{X_{n+1} \in B \mid I_{B}\left(X_{1}\right), \ldots, I_{B}\left(X_{n}\right)\right\}
$$

where $I_{B}\left(X_{i}\right)$ denotes the indicator of the set $\left\{X_{i} \in B\right\}$. Roughly speaking, $a_{n}^{*}(B)$ is the conditional probability that the next observation falls in $B$ given only the history of $B$ in the previous observations. Suppose that the random variable $\mu(B)$ has an absolutely continuous distribution (with respect to Lebesgue measure) for those $B \in \mathcal{D}$ satisfying $0<P\left(X_{1} \in B\right)<1$. Then, for fixed $B \in \mathcal{D}$,

$$
\sqrt{n}\left\{\mu_{n}(B)-a_{n}(B)\right\} \stackrel{P}{\longrightarrow} 0 \Longleftrightarrow \sqrt{n}\left\{a_{n}(B)-a_{n}^{*}(B)\right\} \stackrel{P}{\longrightarrow} 0 .
$$

In addition, under some assumptions on the empirical processes $W_{n}=\sqrt{n}\left(\mu_{n}-\mu\right)$ (satisfied in several real situations), one obtains

$$
\sqrt{n}\left\|\mu_{n}-a_{n}\right\| \stackrel{P}{\longrightarrow} 0 \Longleftrightarrow \sqrt{n}\left\{a_{n}(B)-a_{n}^{*}(B)\right\} \stackrel{P}{\longrightarrow} 0 \text { for each } B \in \mathcal{D} .
$$


However, $\sqrt{n}\left\{a_{n}(B)-a_{n}^{*}(B)\right\}$ may fail to converge to 0 in probability even if $\mu(B)$ has an absolutely continuous distribution; see Example 9.

We finally mention a result (Theorem 10) which, though in the spirit of this paper, is quite different from those described above. Such a result has been inspired by [22]. Let $S=\{0,1\}$ and $\mathcal{C}$ the Borel $\sigma$-field on $[0,1]$. For $C \in \mathcal{C}$, define

$$
\pi_{n}(C)=P\left(\mu_{n}\{1\} \in C\right) \quad \text { and } \quad \pi_{n}^{*}(C)=P\left(a_{n}\{1\} \in C\right)
$$

and denote by $\rho$ the bounded Lipschitz metric between probability measures on $\mathcal{C}$. Then,

$$
\rho\left(\pi_{n}, \pi_{n}^{*}\right) \leq \frac{1}{n}\left(1+\frac{c}{3}\right)
$$

provided the limit frequency $\mu\{1\}$ has an absolutely continuous distribution with Lipschitz density $f$. Here, $c$ is the Lipschitz constant of $f$. This rate of convergence can not be improved.

\section{Motivations}

There are various (non-independent) reasons for investigating how close $\mu_{n}$ and $a_{n}$ are. We now list a few of them under the assumption that

$$
(\Omega, \mathcal{A})=\left(S^{\infty}, \mathcal{B}^{\infty}\right), \quad X_{n}=n \text {-th coordinate projection, } \quad \mathcal{G}=\mathcal{G}^{X} .
$$

Most remarks, however, apply to any filtration $\mathcal{G}$ which makes $X$ adapted.

Similarly, in most of the subsequent comments, $\|\cdot\|$ could be replaced by some other distance $\rho$ between probability measures. For instance, in [10], the asymptotics of $\rho\left(\mu_{n}, a_{n}\right)$ is taken into account with $\rho$ the bounded Lipschitz metric and $\rho$ the Wasserstein distance.

For a general background of Bayesian nonparametrics, often mentioned in what follows, we refer to [18]-[19]; see also [11].

2.1. Bayesian predictive inference. In a number of frameworks, mainly in Bayesian nonparametrics and discrete time filtering, one main goal is to evaluate $a_{n}$. Quite frequently, however, the latter can not be obtained in closed form. For some nonparametric priors, for instance, no closed form expression of $a_{n}$ is known. In these situations, there are essentially two ways out: to compute $a_{n}$ numerically (MCMC) or to estimate it by the available data. If we take the second route, and if data are exchangeable or conditionally identically distributed, $\mu_{n}$ is a reasonable estimate of $a_{n}$. Then, the asymptotic behavior of the error $\mu_{n}-a_{n}$ plays a role. In a sense, this is the basic reason for investigating $\left\|\mu_{n}-a_{n}\right\|$.

2.2. Bayesian consistency. In the spirit of Subsection 2.1, with $\mu_{n}$ regarded as an estimate of $a_{n}$, it makes sense to say that $\mu_{n}$ is consistent if $\left\|\mu_{n}-a_{n}\right\| \rightarrow 0$ a.s. or in probability. In this brief discussion, to fix ideas, we focus on a.s. convergence.

Suppose $X$ is exchangeable. Let $\mathcal{P}$ be the set of all probability measures on $\mathcal{B}$ and $\mu$ the random probability measure on $\mathcal{B}$ introduced in Section 1 . For each $\nu \in \mathcal{P}$, let $P_{\nu}$ denote the probability measure on $\mathcal{B}^{\infty}$ which makes $X$ i.i.d. with common distribution $\nu$. By de Finetti's theorem, conditionally on $\mu$, the sequence $X$ is i.i.d. with common distribution $\mu$; see e.g. [1]. It follows that

$$
P(\cdot)=\int_{\mathcal{P}} P_{\nu}(\cdot) \pi(d \nu)
$$


where $\pi$ is the probability distribution of $\mu$. Such a $\pi$ is usually called the prior distribution.

In the standard approach to consistency, after Diaconis and Freedman [13], the asymptotic behavior of any statistical procedure is investigated under $P_{\nu}$ for each $\nu \in \mathcal{P}$. The procedure is consistent provided it behaves properly for each $\nu \in \mathcal{P}$ (or at least for each $\nu$ in some known subset of $\mathcal{P}$ ); see e.g. [18], [19] and references therein. In particular, $\mu_{n}$ is a consistent estimate of $a_{n}$ if

$$
P_{\nu}\left(\left\|\mu_{n}-a_{n}\right\| \rightarrow 0\right)=1 \quad \text { for each } \nu \in \mathcal{P} .
$$

A different point of view is taken in this paper. Indeed, $\left\|\mu_{n}-a_{n}\right\|$ is investigated under $P$ and $\mu_{n}$ is a consistent estimate of $a_{n}$ if

$$
P\left(\left\|\mu_{n}-a_{n}\right\| \rightarrow 0\right)=1 .
$$

In a sense, in the first approach, consistency of Bayesian procedures is evaluated from a frequentistic point of view. Regarding $\mathcal{P}$ as a parameter space, in fact, $\mu_{n}$ is demanded to approximate $a_{n}$ for each possible value of the parameter $\nu$. This request is certainly admissible. Furthermore, the first notion of consistency is technically stronger than the second. On the other hand, it is not so clear why a Bayesian inferrer should take a frequentistic point of view. Even if $P$ is a mixture of $\left\{P_{\nu}: \nu \in \mathcal{P}\right\}$, when dealing with $X$ the relevant probability measure is $P$ and not $P_{\nu}$. Furthermore, according to de Finetti, any probability statement should concern "observable" facts, while $P_{\nu}$ is conditional on the "unobservable" fact $\mu=\nu$. Thus, according to us, the second approach to consistency is in line with the foundations of Bayesian statistics. A similar opinion is in [10] and [16].

2.3. Frequentistic approximation of Bayesian procedures. In Subsection $2.1, \mu_{n}$ is viewed as an estimate of $a_{n}$. A similar view, developed in [10], is to regard $\mu_{n}$ as a frequentistic approximation of the Bayesian procedure $a_{n}$. For instance, such an approximation makes sense within the empirical Bayes approach, where the orthodox Bayesian reasoning is combined in various ways with frequentistic elements; see e.g. [15] and [25]. We also note that, historically, one reason for introducing exchangeability (possibly, the main reason) was to justify observed frequencies as predictors of future events; see [9] and [28]. In this sense, to focus on $\left\|\mu_{n}-a_{n}\right\|$ is in line with de Finetti's ideas.

2.4. Predictive distributions of exchangeable sequences. If $X$ is exchangeable, just very little is known on the general form of $a_{n}$ for given $n$; see e.g. [16]. Indeed, a representation theorem for $a_{n}$ would be a major breakthrough. Failing the latter, to fix the asymptotic behavior of $\left\|\mu_{n}-a_{n}\right\|$ contributes to fill the gap.

2.5. Empirical processes for non-ergodic data. Slightly abusing terminology, say that $X$ is ergodic if $P$ is $0-1$ valued on the sub- $\sigma$-field

$$
\sigma\left(\limsup _{n} \mu_{n}(B): B \in \mathcal{B}\right) \text {. }
$$

In real problems, $X$ is often non-ergodic. Most stationary sequences, for instance, fail to be ergodic. Or else, an exchangeable sequence is ergodic if and only if is i.i.d. Now, if $X$ is i.i.d., the empirical process is defined as $G_{n}=\sqrt{n}\left(\mu_{n}-\mu_{0}\right)$ where $\mu_{0}$ is the probability distribution of $X_{1}$. But this definition has various drawbacks when $X$ is not ergodic; see [6]. In fact, unless $X$ is i.i.d., the probability distribution of $X$ 
is not determined by that of $X_{1}$. More importantly, if $G_{n}$ converges in distribution in $l^{\infty}(\mathcal{D})$ (the metric space $l^{\infty}(\mathcal{D})$ is recalled before Corollary 8) then

$$
\left\|\mu_{n}-\mu_{0}\right\|=n^{-1 / 2}\left\|G_{n}\right\| \stackrel{P}{\longrightarrow} 0 .
$$

But $\left\|\mu_{n}-\mu_{0}\right\|$ typically fails to converge to 0 in probability when $X$ is not ergodic. Thus, empirical processes for non-ergodic data should be defined in some different way. At least in the exchangeable case, a meaningful option is to center $\mu_{n}$ by $a_{n}$, namely, to let $G_{n}=\sqrt{n}\left(\mu_{n}-a_{n}\right)$.

\section{Assumptions}

Let $\mathcal{D} \subset \mathcal{B}$. To avoid measurability problems, $\mathcal{D}$ is assumed to be countably determined. This means that there is a countable subclass $\mathcal{D}_{0} \subset \mathcal{D}$ such that

$$
\|\alpha-\beta\|=\sup _{B \in \mathcal{D}_{0}}|\alpha(B)-\beta(B)| \text { for all probability measures } \alpha, \beta \text { on } \mathcal{B} .
$$

A sufficient condition is that there is a countable subclass $\mathcal{D}_{0} \subset \mathcal{D}$ such that, for each $B \in \mathcal{D}$ and each probability measure $\alpha$ on $\mathcal{B}$, one obtains

$$
\lim _{n} \alpha\left(B \Delta B_{n}\right)=0 \quad \text { for some sequence } B_{n} \in \mathcal{D}_{0} .
$$

Most classes $\mathcal{D}$ involved in applications are countably determined. For instance, $\mathcal{D}=\mathcal{B}$ is countably determined (for $\mathcal{B}$ is countably generated). Or else, if $S=\mathbb{R}^{k}$, then $\mathcal{D}=\{$ closed convex sets $\}, \mathcal{D}=\{$ half spaces $\}, \mathcal{D}=\{$ closed balls $\}$ and

$$
\mathcal{D}=\left\{\left(-\infty, t_{1}\right] \times \ldots \times\left(-\infty, t_{k}\right]:\left(t_{1}, \ldots, t_{k}\right) \in \mathbb{R}^{k}\right\}
$$

are countably determined.

We next recall the notion of conditionally identically distributed (c.i.d.) random variables. The sequence $X$ is c.i.d. with respect to $\mathcal{G}$ if it is $\mathcal{G}$-adapted and

$$
P\left(X_{k} \in \cdot \mid \mathcal{G}_{n}\right)=P\left(X_{n+1} \in \cdot \mid \mathcal{G}_{n}\right) \quad \text { a.s. for all } k>n \geq 0 .
$$

Roughly speaking, at each time $n \geq 0$, the future observations $\left(X_{k}: k>n\right)$ are identically distributed given the past $\mathcal{G}_{n}$. When $\mathcal{G}=\mathcal{G}^{X}$, the filtration $\mathcal{G}$ is not mentioned at all and $X$ is just called c.i.d. Then, $X$ is c.i.d. if and only if

$$
\left(X_{1}, \ldots, X_{n}, X_{n+2}\right) \sim\left(X_{1}, \ldots, X_{n}, X_{n+1}\right) \text { for all } n \geq 0 .
$$

Exchangeable sequences are c.i.d., for they meet (2), while the converse is not true. Indeed, $X$ is exchangeable if and only if it is stationary and c.i.d. We refer to [4] for more on c.i.d. sequences. Here, it suffices to mention the strong law of large numbers and some of its consequences.

If $X$ is c.i.d., there is a random probability measure $\mu$ on $\mathcal{B}$ satisfying

$$
\mu_{n}(B) \stackrel{a . s_{.}}{\longrightarrow} \mu(B) \text { for every } B \in \mathcal{B} .
$$

As a consequence, if $X$ is c.i.d. with respect to $\mathcal{G}$, for each $n \geq 0$ and $B \in \mathcal{B}$ one obtains

$$
\begin{aligned}
E\left\{\mu(B) \mid \mathcal{G}_{n}\right\}= & \lim _{m} E\left\{\mu_{m}(B) \mid \mathcal{G}_{n}\right\}=\lim _{m} \frac{1}{m} \sum_{k=n+1}^{m} P\left(X_{k} \in B \mid \mathcal{G}_{n}\right) \\
& =P\left(X_{n+1} \in B \mid \mathcal{G}_{n}\right)=a_{n}(B) \text { a.s. }
\end{aligned}
$$

In particular, $a_{n}(B)=E\left\{\mu(B) \mid \mathcal{G}_{n}\right\} \stackrel{a . s}{\longrightarrow} \mu(B)$ so that $\mu_{n}(B)-a_{n}(B) \stackrel{a . s_{.}}{\longrightarrow} 0$. 
From now on, $X$ is c.i.d. with respect to $\mathcal{G}$. In particular, $X$ is identically distributed and $\mu_{0}$ denotes the probability distribution of $X_{1}$. We also let

$$
W_{n}=\sqrt{n}\left(\mu_{n}-\mu\right) .
$$

Note that, if $X$ is i.i.d., then $\mu=\mu_{0}$ a.s. and $W_{n}$ reduces to the usual empirical process.

\section{Results}

Our results can be sorted into three subsections.

4.1. Two general criterions. Since $a_{n}(B)=E\left\{\mu(B) \mid \mathcal{G}_{n}\right\}$ a.s. and $\mathcal{D}$ is countably determined, one obtains

$$
\begin{gathered}
\left\|\mu_{n}-a_{n}\right\|=\sup _{B \in \mathcal{D}_{0}}\left|\mu_{n}(B)-a_{n}(B)\right| \\
=\sup _{B \in \mathcal{D}_{0}}\left|E\left\{\mu_{n}(B)-\mu(B) \mid \mathcal{G}_{n}\right\}\right| \leq E\left\{\left\|\mu_{n}-\mu\right\| \mid \mathcal{G}_{n}\right\} \quad \text { a.s. }
\end{gathered}
$$

This simple inequality has some nice consequences. Recall that $\mathcal{D}$ is a universal Glivenko-Cantelli class if $\left\|\mu_{n}-\mu_{0}\right\| \stackrel{a . s}{\longrightarrow} 0$ whenever $X$ is i.i.d.; see e.g. [14], [17], [27].

Theorem 1. ([3] and [5]). Suppose $\mathcal{D}$ is countably determined and $X$ is c.i.d. with respect to $\mathcal{G}$. Then,

(i) $\left\|\mu_{n}-a_{n}\right\| \stackrel{a . s .}{\longrightarrow} 0$ if $\left\|\mu_{n}-\mu\right\| \stackrel{a . s .}{\longrightarrow} 0$ and $\left\|\mu_{n}-a_{n}\right\| \stackrel{P}{\longrightarrow} 0$ if $\left\|\mu_{n}-\mu\right\| \stackrel{P}{\longrightarrow} 0$. In particular, $\left\|\mu_{n}-a_{n}\right\| \stackrel{\text { a.s. }}{\longrightarrow} 0$ provided $X$ is exchangeable, $\mathcal{G}=\mathcal{G}^{X}$ and $\mathcal{D}$ is a universal Glivenko-Cantelli class.

(ii) $r_{n}\left\|\mu_{n}-a_{n}\right\| \stackrel{P}{\longrightarrow} 0$ whenever the constants $r_{n}$ satisfy $r_{n} / \sqrt{n} \rightarrow 0$ and $\sup _{n} E\left\{\left\|W_{n}\right\|^{p}\right\}<\infty$ for some $p \geq 1$.

Proof. Since $\left\|\mu_{n}-\mu\right\| \leq 1$, if $\left\|\mu_{n}-\mu\right\| \stackrel{a . s}{\longrightarrow} 0$ then

$$
\left\|\mu_{n}-a_{n}\right\| \leq E\left\{\left\|\mu_{n}-\mu\right\| \mid \mathcal{G}_{n}\right\} \stackrel{a . s}{\longrightarrow} 0
$$

because of the martingale convergence theorem in the version of [8]. Similarly, $\left\|\mu_{n}-\mu\right\| \stackrel{P}{\longrightarrow} 0$ implies $E\left\{\left\|\mu_{n}-\mu\right\| \mid \mathcal{G}_{n}\right\} \stackrel{P}{\longrightarrow} 0$ by an obvious argument based on subsequences. Next, let $X$ be exchangeable. By de Finetti's theorem, conditionally on $\mu$, the sequence $X$ is i.i.d. with common distribution $\mu$. If $\mathcal{D}$ is a universal Glivenko-Cantelli class, it follows that

$$
P\left(\left\|\mu_{n}-\mu\right\| \rightarrow 0\right)=\int P\left\{\left\|\mu_{n}-\mu\right\| \rightarrow 0 \mid \mu\right\} d P=\int 1 d P=1 .
$$

This concludes the proof of (i). As to (ii), just note that

$$
\begin{gathered}
E\left\{\left(r_{n}\left\|\mu_{n}-a_{n}\right\|\right)^{p}\right\} \leq r_{n}^{p} E\left\{E\left\{\left\|\mu_{n}-\mu\right\| \mid \mathcal{G}_{n}\right\}^{p}\right\} \\
\leq r_{n}^{p} E\left\{\left\|\mu_{n}-\mu\right\|^{p}\right\}=\left(r_{n} / \sqrt{n}\right)^{p} E\left\{\left\|W_{n}\right\|^{p}\right\} .
\end{gathered}
$$

While Theorem 1 is essentially known (the proof has been provided for completeness only) the next result is new. 
Theorem 2. Suppose $\mathcal{D}$ is countably determined and $X$ is c.i.d. with respect to $\mathcal{G}$. Fix the constants $r_{n}>0$ and define

$$
M_{k}=\sup _{n \geq k} r_{n}\left\|\mu_{n}-\mu\right\| .
$$

If $E\left(M_{k}\right)<\infty$ for some $k$, then

$$
\limsup _{n} r_{n}\left\|\mu_{n}-a_{n}\right\| \leq \underset{n}{\limsup } r_{n}\left\|\mu_{n}-\mu\right\|<\infty \quad \text { a.s. }
$$

Moreover, if $X$ is exchangeable, then $E\left(M_{k}\right)<\infty$ for some $k$ whenever

(iii) $r_{n}=\frac{\sqrt{n}}{(\log n)^{1 / c}}$ and $\sup _{n} E\left\{\left\|W_{n}\right\|^{p}\right\}<\infty$ for some $p>1$ and $0<c<p$;

(iv) $r_{n}=\sqrt{\frac{n}{\log \log n}}$ and

$$
\sup _{n} E\left\{\exp \left(u\left\|W_{n}\right\|\right)\right\} \leq a \exp \left(b u^{2}\right) \quad \text { for all } u>0 \text { and some } a, b>0 .
$$

Proof. Fix $j \geq k$. Since $E\left(M_{j}\right) \leq E\left(M_{k}\right)<\infty$, then

$\limsup _{n} r_{n}\left\|\mu_{n}-a_{n}\right\| \leq \underset{n}{\limsup } E\left\{r_{n}\left\|\mu_{n}-\mu\right\| \mid \mathcal{G}_{n}\right\} \leq \underset{n}{\lim \sup } E\left\{M_{j} \mid \mathcal{G}_{n}\right\}=M_{j} \quad$ a.s.

where the last equality is due to the martingale convergence theorem. Hence,

$$
\limsup _{n} r_{n}\left\|\mu_{n}-a_{n}\right\| \leq \inf _{j \geq k} M_{j}=\limsup _{n} r_{n}\left\|\mu_{n}-\mu\right\| \quad \text { a.s. }
$$

Further, $E\left(M_{k}\right)<\infty$ obviously implies $\lim \sup _{n} r_{n}\left\|\mu_{n}-\mu\right\| \leq M_{k}<\infty$ a.s.

Next, suppose $X$ exchangeable. Then,

$$
S_{n}=n\left\|\mu_{n}-\mu\right\|=\sqrt{n}\left\|W_{n}\right\|
$$

is a submartingale with respect to the filtration $\mathcal{U}_{n}=\sigma\left[\mathcal{G}_{n}^{X} \cup \sigma(\mu)\right]$. In fact,

$$
\begin{aligned}
& (n+1) E\left\{\mu_{n+1}(B) \mid \mathcal{U}_{n}\right\}=n \mu_{n}(B)+P\left\{X_{n+1} \in B \mid \mathcal{U}_{n}\right\} \\
& =n \mu_{n}(B)+P\left\{X_{n+1} \in B \mid \sigma(\mu)\right\}=n \mu_{n}(B)+\mu(B) \text { a.s. }
\end{aligned}
$$

Therefore,

$$
E\left(S_{n+1} \mid \mathcal{U}_{n}\right) \geq(n+1) \sup _{B \in \mathcal{D}}\left|E\left\{\mu_{n+1}(B) \mid \mathcal{U}_{n}\right\}-\mu(B)\right|=n \sup _{B \in \mathcal{D}}\left|\mu_{n}(B)-\mu(B)\right|=S_{n} \quad \text { a.s. }
$$

(iii) Let $r_{n}=\frac{\sqrt{n}}{(\log n)^{1 / c}}$ and $\sup _{n} E\left\{\left\|W_{n}\right\|^{p}\right\}<\infty$, where $p>1$ and $0<c<p$. Then,

$$
E\left(M_{3}^{p}\right)=E\left\{\left(\sup _{n \geq 1} \max _{2^{n}<j \leq 2^{(n+1)}} r_{j}\left\|\mu_{j}-\mu\right\|\right)^{p}\right\} \leq \sum_{n=1}^{\infty} E\left\{\max _{2^{n}<j \leq 2^{(n+1)}} r_{j}^{p}\left\|\mu_{j}-\mu\right\|^{p}\right\} .
$$

If $2^{n}<j \leq 2^{(n+1)}$, then

$$
r_{j}\left\|\mu_{j}-\mu\right\|=j^{-1 / 2}(\log j)^{-1 / c} S_{j} \leq\left(2^{n}\right)^{-1 / 2}\left(\log 2^{n}\right)^{-1 / c} S_{j} .
$$


By such inequality and since $\left(S_{j}\right)$ is a submartingale, one obtains

$$
\begin{gathered}
E\left(M_{3}^{p}\right) \leq \sum_{n}\left(2^{n}\right)^{-p / 2}\left(\log 2^{n}\right)^{-p / c} E\left\{\max _{j \leq 2^{(n+1)}} S_{j}^{p}\right\} \\
\leq(p /(p-1))^{p} \sum_{n}\left(2^{n}\right)^{-p / 2}\left(\log 2^{n}\right)^{-p / c} E\left\{S_{2^{(n+1)}}^{p}\right\} \\
=(p /(p-1))^{p} 2^{p / 2} \sum_{n}\left(\log 2^{n}\right)^{-p / c} E\left\{\left\|W_{2^{(n+1)}}\right\|^{p}\right\} \\
\left.\leq \sup _{j} E\left\{\left\|W_{j}\right\|^{p}\right\}\right)(p /(p-1))^{p} 2^{p / 2}(\log 2)^{-p / c} \sum_{n} n^{-p / c}<\infty .
\end{gathered}
$$

(iv) Let $r_{n}=\sqrt{\frac{n}{\log \log n}}$ and $\sup _{n} E\left\{\exp \left(u\left\|W_{n}\right\|\right)\right\} \leq a \exp \left(b u^{2}\right)$ for all $u>0$ and some $a, b>0$. We aim to prove that

$P\left(M_{4}>t\right) \leq c \exp \left(-v t^{2}\right) \quad$ for large $t$ and suitable constants $c, v>0$.

In this case, in fact, $E\left(M_{4}\right)=\int_{0}^{\infty} P\left(M_{4}>t\right) d t<\infty$.

First note that

$$
\begin{gathered}
P\left(M_{4}>t\right)=P\left(\bigcup_{n \geq 1}\left\{\max _{3^{n}<j \leq 3^{(n+1)}} r_{j}\left\|\mu_{j}-\mu\right\|>t\right\}\right) \leq \sum_{n=1}^{\infty} P\left(\max _{j \leq 3^{(n+1)}} S_{j}>m_{n} t\right) \\
\text { where } m_{n}=\sqrt{3^{n} \log \log 3^{n}}=\sqrt{3^{n}(\log n+\log \log 3)} .
\end{gathered}
$$

Let $\theta>0$. On noting that $\exp \left(\theta S_{n}\right)$ is still a submartingale, one also obtains

$$
\begin{gathered}
P\left(\max _{j \leq 3^{(n+1)}} S_{j}>m_{n} t\right)=P\left(\max _{j \leq 3^{(n+1)}} \exp \left(\theta S_{j}\right)>\exp \left(\theta m_{n} t\right)\right) \\
\leq \exp \left(-\theta m_{n} t\right) E\left\{\exp \left(\theta S_{3^{(n+1)}}\right)\right\} \\
=\exp \left(-\theta m_{n} t\right) E\left\{\exp \left(\theta \sqrt{3^{(n+1)}}\left\|W_{3^{(n+1)}}\right\|\right)\right\} \\
\leq a \exp \left(-\theta m_{n} t+\theta^{2} b 3^{(n+1)}\right) .
\end{gathered}
$$

The minimum over $\theta$ is attained at $\theta=\frac{m_{n} t}{6 b 3^{n}}$. Thus,

$$
P\left(\max _{j \leq 3^{(n+1)}} S_{j}>m_{n} t\right) \leq a \exp \left(\frac{-m_{n}^{2} t^{2}}{12 b 3^{n}}\right)=a \exp \left(\frac{-t^{2} \log \log 3}{12 b}\right) n^{-t^{2} / 12 b} .
$$

If $t \geq \sqrt{24 b}$, then $t^{2}>12 b$ and $\frac{t^{2}}{t^{2}-12 b} \leq 2$. Thus, one finally obtains

$$
\begin{gathered}
P\left(M_{4}>t\right) \leq a \exp \left(\frac{-t^{2} \log \log 3}{12 b}\right) \sum_{n} n^{-t^{2} / 12 b} \\
\leq a \exp \left(\frac{-t^{2} \log \log 3}{12 b}\right) \frac{t^{2}}{t^{2}-12 b} \\
\leq 2 a \exp \left(\frac{-t^{2} \log \log 3}{12 b}\right) \text { for every } t \geq \sqrt{24 b} .
\end{gathered}
$$

Some remarks are in order. In the sequel, if $\alpha$ and $\beta$ are measures on a $\sigma$-field $\mathcal{E}$, we write $\alpha \ll \beta$ to mean that $\alpha$ is absolutely continuous with respect to $\beta$, namely, $\alpha(A)=0$ whenever $A \in \mathcal{E}$ and $\beta(A)=0$. 
- Sometimes, the condition of Theorem 1-(i) is necessary as well, namely, $\left\|\mu_{n}-a_{n}\right\| \stackrel{a . s}{\longrightarrow} 0$ if and only if $\left\|\mu_{n}-\mu\right\| \stackrel{a . s}{\longrightarrow} 0$. For instance, this happens when $\mathcal{G}=\mathcal{G}^{X}$ and $\mu \ll \lambda$ a.s., where $\lambda$ is a (non-random) $\sigma$-finite measure on $\mathcal{B}$. In this case, in fact, $\left\|a_{n}-\mu\right\| \stackrel{a . s}{\longrightarrow} 0$ by [7, Theorem 1].

- Several examples of universal Glivenko-Cantelli classes are available; see [14], [17], [27] and references therein. Moreover, for many choices of $\mathcal{D}$ and $p$ there is a universal constant $c(p)$ such that $\sup _{n} E\left\{\left\|W_{n}\right\|^{p}\right\} \leq c(p)$ provided $X$ is i.i.d.; see e.g. [27, Sect. 2.14.1-2.14.2]. For such $\mathcal{D}$ and $p$, de Finetti's theorem yields $\sup _{n} E\left\{\left\|W_{n}\right\|^{p}\right\} \leq c(p)$ even if $X$ is exchangeable. In fact, conditionally on $\mu$, the sequence $X$ is i.i.d. with common distribution $\mu$. Hence, $E\left\{\left\|W_{n}\right\|^{p} \mid \mu\right\} \leq c(p)$ a.s. for all $n$. By the same argument, if there are $a, b>0$ such that

$$
\sup _{n} E\left\{\exp \left(u\left\|W_{n}\right\|\right)\right\} \leq a \exp \left(b u^{2}\right) \quad \text { for all } u>0 \text { if } X \text { is i.i.d., }
$$

such inequality is still true (with the same $a$ and $b$ ) if $X$ is exchangeable.

- A straightforward consequence of the law of iterated logarithm is that convergence in probability can not be replaced by a.s. convergence in Theorem 1-(ii). Take in fact $r_{n}=\sqrt{\frac{n}{\log \log n}}, \mathcal{G}=\mathcal{G}^{X}$ and $X$ i.i.d. Then, for each $B \in \mathcal{D}$, the law of iterated logarithm yields

$$
\begin{gathered}
\limsup _{n} r_{n}\left\|\mu_{n}-a_{n}\right\| \geq \underset{n}{\limsup } r_{n}\left\{\mu_{n}(B)-a_{n}(B)\right\} \\
=\limsup _{n} \frac{\sum_{i=1}^{n}\left\{I_{B}\left(X_{i}\right)-\mu_{0}(B)\right\}}{\sqrt{n \log \log n}}=\sqrt{2 \mu_{0}(B)\left(1-\mu_{0}(B)\right)} \text { a.s. }
\end{gathered}
$$

- Let $\mathcal{D}$ be countably determined, $X$ exchangeable and $\mathcal{G}=\mathcal{G}^{X}$. In view of Theorem 2, for $r_{n}\left\|\mu_{n}-a_{n}\right\| \stackrel{a . s}{\longrightarrow} 0$, it suffices that $\sup _{n} E\left\{\left\|W_{n}\right\|^{p}\right\}<\infty$ and $\frac{r_{n}(\log n)^{1 / c}}{\sqrt{n}} \rightarrow 0$, for some $p>1$ and $0<c<p$, or that $E\left\{\exp \left(u\left\|W_{n}\right\|\right)\right\}$ can be estimated as in (iv) and $r_{n} \sqrt{\frac{\log \log n}{n}} \rightarrow 0$. For instance,

$$
\sqrt{\frac{n}{\log n}}\left\|\mu_{n}-a_{n}\right\| \stackrel{a . s s}{\longrightarrow} 0
$$

whenever $\sup _{n} E\left\{\left\|W_{n}\right\|^{p}\right\}<\infty$ for some $p>2$. Another example is provided by Corollary 3 . To state it, a definition is to be recalled.

Say that $\mathcal{D}$ is a Vapnik-Cervonenkis class, or simply a VC-class, if

$$
\text { card }\{B \cap I: B \in \mathcal{D}\}<2^{n}
$$

for some integer $n \geq 1$ and all subsets $I \subset S$ with $\operatorname{card}(I)=n$; see e.g. [14], [17], [21], [27]. In other terms, the power set of $I$ can not be written as $\{B \cap I: B \in \mathcal{D}\}$ for each collection $I$ of $n$ points from $S$. As noted in Section 1, VC-classes are instrumental to empirical processes and statistical learning. If $S=\mathbb{R}^{k}$, for instance, $\mathcal{D}=\left\{\left(-\infty, t_{1}\right] \times \ldots \times\left(-\infty, t_{k}\right]:\left(t_{1}, \ldots, t_{k}\right) \in \mathbb{R}^{k}\right\}, \mathcal{D}=\{$ half spaces $\}$ and $\mathcal{D}=\{$ closed balls $\}$ are (countably determined) VC-classes. 
Corollary 3. Let $\mathcal{D}$ be a countably determined VC-class. If $X$ is exchangeable and $\mathcal{G}=\mathcal{G}^{X}$, then

$$
\limsup _{n} \sqrt{\frac{n}{\log \log n}}\left\|\mu_{n}-a_{n}\right\| \leq \sqrt{2 \sup _{B \in \mathcal{D}} \mu(B)(1-\mu(B))} \quad \text { a.s. }
$$

Proof. Just note that, if $X$ is i.i.d. and $\mathcal{D}$ is a countably determined VC-class, then $E\left\{\exp \left(u\left\|W_{n}\right\|\right)\right\}$ can be estimated as in Theorem 2-(iv) and

$$
\limsup _{n} \sqrt{\frac{n}{\log \log n}}\left\|\mu_{n}-\mu_{0}\right\|=\sqrt{2 \sup _{B \in \mathcal{D}} \mu_{0}(B)\left(1-\mu_{0}(B)\right)} \text { a.s. }
$$

See e.g. [14, Sect. 9.5], [21, Corollary 2.4] and [27, page 246].

We finally give a couple of examples concerning Theorem 1.

Example 4. Let $\mathcal{D}=\mathcal{B}$. If $X$ is i.i.d., then $\left\|\mu_{n}-\mu_{0}\right\| \stackrel{a . s}{\longrightarrow} 0$ if and only if $\mu_{0}$ is discrete. By de Finetti's theorem, it follows that $\left\|\mu_{n}-\mu\right\| \stackrel{a . s}{\longrightarrow} 0$ whenever $X$ is exchangeable and $\mu$ is a.s. discrete. Thus, under such assumptions and $\mathcal{G}=\mathcal{G}^{X}$, Theorem 1-(i) implies $\left\|\mu_{n}-a_{n}\right\| \stackrel{a . s}{\longrightarrow} 0$. This result has a possible practical interest in Bayesian nonparametrics. As noted in Section 1, in fact, most nonparametric priors are such that $\mu$ is a.s. discrete.

Example 5. Let $S=\mathbb{R}^{k}$ and $\mathcal{D}=\{$ closed convex sets $\}$. If $X$ is i.i.d. and $\mu_{0} \ll \lambda$, where $\lambda$ is a $\sigma$-finite product measure on $\mathcal{B}$, then $\left\|\mu_{n}-\mu_{0}\right\| \stackrel{a . s .}{\longrightarrow} 0$; see $[17$, page 198]. Applying Theorem 1-(i) again, one obtains $\left\|\mu_{n}-a_{n}\right\| \stackrel{a . s}{\longrightarrow} 0$ provided $X$ is exchangeable, $\mathcal{G}=\mathcal{G}^{X}$ and $\mu \ll \lambda$ a.s. While "morally true", this argument does not work for $\mathcal{D}=\{$ Borel convex sets $\}$ since the latter choice of $\mathcal{D}$ is not countably determined.

4.2. The dominated case. In the sequel, as in Section 2, it is convenient to work on the coordinate space. Accordingly, from now on, we let

$$
(\Omega, \mathcal{A})=\left(S^{\infty}, \mathcal{B}^{\infty}\right), \quad X_{n}=n \text {-th coordinate projection, } \quad \mathcal{G}=\mathcal{G}^{X} .
$$

Further, $Q$ is a probability measure on $(\Omega, \mathcal{A})$ and

$$
b_{n}(\cdot)=Q\left(X_{n+1} \in \cdot \mid \mathcal{G}_{n}\right)
$$

is the predictive measure under $Q$. We say that $Q$ is a Ferguson-Dirichlet law if

$$
b_{n}(\cdot)=\frac{c Q\left(X_{1} \in \cdot\right)+n \mu_{n}(\cdot)}{c+n}, \quad Q \text {-a.s. for some constant } c>0 .
$$

If $P \ll Q$, the asymptotic behavior of $\left\|\mu_{n}-a_{n}\right\|$ under $P$ should be affected by that of $\left\|\mu_{n}-b_{n}\right\|$ under $Q$. This (rough) idea is realized by the next result.

Theorem 6. (Theorems 1 and $\mathbf{2}$ of [5]). Suppose $\mathcal{D}$ is countably determined, $X$ is c.i.d., and $P \ll Q$. Then,

$$
\sqrt{n}\left\|\mu_{n}-a_{n}\right\| \stackrel{P}{\longrightarrow} 0
$$

whenever $\sqrt{n}\left\|\mu_{n}-b_{n}\right\| \stackrel{Q}{\longrightarrow} 0$ and the sequence $\left(W_{n}\right)$ is uniformly integrable under both $P$ and $Q$. In addition,

$$
n\left\|\mu_{n}-a_{n}\right\| \quad \text { converges a.s. to a finite limit }
$$


provided $Q$ is a Ferguson-Dirichlet law, $\sup _{n} E_{Q}\left\{\left\|W_{n}\right\|^{2}\right\}<\infty$, and

$$
\sup _{n} n\left\{E_{Q}\left(f^{2}\right)-E_{Q}\left\{E_{Q}\left(f \mid \mathcal{G}_{n}\right)^{2}\right\}\right\}<\infty \quad \text { where } f=d P / d Q \text {. }
$$

To make Theorem 6 effective, the condition $P \ll Q$ should be given a simple characterization. This happens at least when $S$ is finite.

As an example, suppose $S=\{0,1\}, X$ exchangeable and $Q$ Ferguson-Dirichlet. Then, for all $n \geq 1$ and $x_{1}, \ldots, x_{n} \in\{0,1\}$,

$$
\begin{aligned}
& P\left(X_{1}=x_{1}, \ldots, X_{n}=x_{n}\right)=\int_{[0,1]} \theta^{k}(1-\theta)^{n-k} \pi_{P}(d \theta), \\
& Q\left(X_{1}=x_{1}, \ldots, X_{n}=x_{n}\right)=\int_{[0,1]} \theta^{k}(1-\theta)^{n-k} \pi_{Q}(d \theta),
\end{aligned}
$$

where $k=\sum_{i=1}^{n} x_{i}$ and $\pi_{P}$ and $\pi_{Q}$ are the probability distributions of $\mu\{1\}$ under $P$ and $Q$. Thus, $P \ll Q$ if and only if $\pi_{P} \ll \pi_{Q}$. In addition, $\pi_{Q}$ is known to be a beta distribution. Let $m$ denote the Lebesgue measure on the Borel $\sigma$-field on $[0,1]$. Since any beta distribution has the same null sets as $m$, one obtains $P \ll Q$ if and only if $\pi_{P} \ll m$. This fact is behind the next result.

Theorem 7. (Corollaries 4 and 5 of [5]). Suppose $S=\{0,1\}$ and $X$ exchangeable. Then, $\sqrt{n}\left(\mu_{n}\{1\}-a_{n}\{1\}\right) \stackrel{P}{\longrightarrow} 0$ whenever the distribution of $\mu\{1\}$ is absolutely continuous. Moreover, $n\left(\mu_{n}\{1\}-a_{n}\{1\}\right)$ converges a.s. (to a finite limit) provided the distribution of $\mu\{1\}$ is absolutely continuous with an almost Lipschitz density.

In Theorem 7 , a real function $f$ on $(0,1)$ is said to be almost Lipschitz in case $x \mapsto f(x) x^{u}(1-x)^{v}$ is Lipschitz on $(0,1)$ for some reals $u, v<1$.

A consequence of Theorem 7 is to be stressed. For each $B \in \mathcal{B}$, define

$\mathcal{G}_{n}^{B}=\sigma\left(I_{B}\left(X_{1}\right), \ldots, I_{B}\left(X_{n}\right)\right) \quad$ and $\quad T_{n}(B)=\sqrt{n}\left\{a_{n}(B)-P\left\{X_{n+1} \in B \mid \mathcal{G}_{n}^{B}\right\}\right\}$.

Also, let $l^{\infty}(\mathcal{D})$ be the set of real bounded functions on $\mathcal{D}$, equipped with uniform distance. In the next result, $W_{n}$ is regarded as a random element of $l^{\infty}(\mathcal{D})$ and convergence in distribution is meant in Hoffmann-Jørgensen's sense; see [27].

Corollary 8. Let $\mathcal{D}$ be countably determined and $X$ exchangeable. Suppose that

(j) $\mu(B)$ has an absolutely continuous distribution for each $B \in \mathcal{D}$ such that $0<P\left(X_{1} \in B\right)<1 ;$

(jj) the sequence $\left(\left\|W_{n}\right\|\right)$ is uniformly integrable;

(jjj) $W_{n}$ converges in distribution, in the space $l^{\infty}(\mathcal{D})$, to a tight limit.

Then,

$$
\sqrt{n}\left\|\mu_{n}-a_{n}\right\| \stackrel{P}{\longrightarrow} 0 \Longleftrightarrow T_{n}(B) \stackrel{P}{\longrightarrow} 0 \text { for each } B \in \mathcal{D} \text {. }
$$

Proof. Let $U_{n}(B)=\sqrt{n}\left\{\mu_{n}(B)-P\left\{X_{n+1} \in B \mid \mathcal{G}_{n}^{B}\right\}\right\}$. Then, $U_{n}(B) \stackrel{P}{\longrightarrow} 0$ for each $B \in \mathcal{D}$. In fact, $U_{n}(B)=0$ a.s. if $P\left(X_{1} \in B\right) \in\{0,1\}$. Otherwise, $U_{n}(B) \stackrel{P}{\longrightarrow} 0$ follows from Theorem 7 , since $\left(I_{B}\left(X_{n}\right)\right)$ is an exchangeable sequence of indicators and $\mu(B)$ has an absolutely continuous distribution. Next, suppose 
$T_{n}(B) \stackrel{P}{\longrightarrow} 0$ for each $B \in \mathcal{D}$. Letting $C_{n}=\sqrt{n}\left(\mu_{n}-a_{n}\right)$, we have to prove that $\left\|C_{n}\right\| \stackrel{P}{\longrightarrow}$ 0. Equivalently, regarding $C_{n}$ as a random element of $l^{\infty}(\mathcal{D})$, we have to prove that $C_{n}(B) \stackrel{P}{\longrightarrow} 0$ for fixed $B \in \mathcal{D}$ and the sequence $\left(C_{n}\right)$ is asymptotically tight; see e.g. $\left[27\right.$, Section 1.5]. Given $B \in \mathcal{D}$, since both $U_{n}(B)$ and $T_{n}(B)$ converge to 0 in probability, then $C_{n}(B)=U_{n}(B)-T_{n}(B) \stackrel{P}{\longrightarrow} 0$. Moreover, since $C_{n}(B)=E\left\{W_{n}(B) \mid \mathcal{G}_{n}\right\}$ a.s., the asymptotic tightness of $\left(C_{n}\right)$ follows from (jj)(jjj); see $\left[4\right.$, Remark 4.4]. Hence, $\left\|C_{n}\right\| \stackrel{P}{\longrightarrow} 0$. Conversely, if $\left\|C_{n}\right\| \stackrel{P}{\longrightarrow} 0$, one trivially obtains

$$
\left|T_{n}(B)\right|=\left|U_{n}(B)-C_{n}(B)\right| \leq\left|U_{n}(B)\right|+\left\|C_{n}\right\| \stackrel{P}{\longrightarrow} 0 \quad \text { for each } B \in \mathcal{D} .
$$

If $X$ is exchangeable, it frequently happens that $\sup _{n} E\left\{\left\|W_{n}\right\|^{2}\right\}<\infty$, which in turn implies condition (jj). Similarly, (jjj) is not unusual. As an example, conditions (jj)-(jjj) hold if $S=\mathbb{R}, \mathcal{D}=\{(-\infty, t]: t \in \mathbb{R}\}$ and $\mu_{0}$ is discrete or $P\left(X_{1}=X_{2}\right)=0$; see $[4$, Theorem 4.5].

Unfortunately, as shown by the next example, $T_{n}(B)$ may fail to converge to 0 in probability even if $\mu(B)$ has an absolutely continuous distribution. This suggests the following general question. In the exchangeable case, in addition to $\mu_{n}(B)$, which further information is required to evaluate $a_{n}(B)$ ? Or at least, are there reasonable conditions for $T_{n}(B) \stackrel{P}{\longrightarrow} 0$ ? Even if intriguing, to our knowledge, such a question does not have a satisfactory answer.

Example 9. Let $S=\mathbb{R}$ and $X_{n}=Y_{n} Z^{-1}$, where $Y_{n}$ and $Z$ are independent real random variables, $Y_{n} \sim N(0,1)$ for all $n$, and $Z$ has an absolutely continuous distribution supported by $[1, \infty)$. Conditionally on $Z$, the sequence $X=\left(X_{1}, X_{2}, \ldots\right)$ is i.i.d. with common distribution $N\left(0, Z^{-2}\right)$. Thus, $X$ is exchangeable and

$$
\begin{gathered}
\mu(B)=P\left(X_{1} \in B \mid Z\right)=f_{B}(Z) \quad \text { a.s. for each } B \in \mathcal{B} \\
\text { where } \quad f_{B}(z)=(2 \pi)^{-1 / 2} z \int_{B} \exp \left(-(x z)^{2} / 2\right) d x \quad \text { for } z \geq 1 .
\end{gathered}
$$

Fix $B \in \mathcal{B}$, with $B \subset[1, \infty)$ and $P\left(X_{1} \in B\right)>0$, and set $C=\{-x: x \in$ $B$. Since $f_{B}=f_{C}$, then $\mu(B)=\mu(C)$ and $a_{n}(B)=a_{n}(C)$ a.s. Further, $\mu(B)$ has an absolutely continuous distribution, for $f_{B}$ is differentiable and $f_{B}^{\prime} \neq 0$. Nevertheless, one between $T_{n}(B)$ and $T_{n}(C)$ does not converge to 0 in probability. Define in fact $g=I_{B}-I_{C}$ and $R_{n}=n^{-1 / 2} \sum_{i=1}^{n} g\left(X_{i}\right)$. Since $\mu(g)=\mu(B)-\mu(C)=$ 0 a.s., then $R_{n}$ converges stably to the kernel $N(0,2 \mu(B))$; see [4, Theorem 3.1]. On the other hand, since $a_{n}(B)=a_{n}(C)$ a.s., one obtains

$$
\begin{gathered}
R_{n}=\sqrt{n}\left\{\mu_{n}(B)-\mu_{n}(C)\right\}=T_{n}(C)-T_{n}(B)+ \\
+\sqrt{n}\left\{\mu_{n}(B)-P\left\{X_{n+1} \in B \mid \mathcal{G}_{n}^{B}\right\}\right\}-\sqrt{n}\left\{\mu_{n}(C)-P\left\{X_{n+1} \in C \mid \mathcal{G}_{n}^{C}\right\}\right\} \quad \text { a.s. }
\end{gathered}
$$

Therefore, if $T_{n}(B) \stackrel{P}{\longrightarrow} 0$ and $T_{n}(C) \stackrel{P}{\longrightarrow} 0$, Theorem 7 implies the contradiction $R_{n} \stackrel{P}{\longrightarrow} 0$.

4.3. Exchangeable sequences of indicators. Let $\mathcal{P}$ be the set of all probability measures on $\mathcal{B}$, equipped with the topology of weak convergence. Since $\mu_{n}$ and $a_{n}$ are $\mathcal{P}$-valued random variables, we can define their probability distributions on the 
Borel $\sigma$-field on $\mathcal{P}$, say $\pi_{n}(\cdot)=P\left(\mu_{n} \in \cdot\right)$ and $\pi_{n}^{*}(\cdot)=P\left(a_{n} \in \cdot\right)$. Another way to compare $\mu_{n}$ and $a_{n}$, different from the one adopted so far, is to focus on $\rho\left(\pi_{n}, \pi_{n}^{*}\right)$ where $\rho$ is a suitable distance between the Borel probability measures on $\mathcal{P}$. In this subsection, we actually take this point of view.

Let $\mathcal{C}$ be the Borel $\sigma$-field on $[0,1]$ and $\rho$ the bounded Lipschitz metric between probability measures on $\mathcal{C}$. We recall that $\rho$ is defined as

$$
\rho\left(\pi, \pi^{*}\right)=\sup _{\phi}\left|\pi(\phi)-\pi^{*}(\phi)\right|
$$

where $\pi$ and $\pi^{*}$ are probability measures on $\mathcal{C}$ and sup is over those functions $\phi$ on $[0,1]$ such that $\phi$ is 1 -Lipschitz and $-1 \leq \phi \leq 1$.

Suppose $S=\{0,1\}$ and $X$ exchangeable. Define $\pi_{n}(C)=P\left(\mu_{n}\{1\} \in C\right)$ and $\pi_{n}^{*}(C)=P\left(a_{n}\{1\} \in C\right)$ for $C \in \mathcal{C}$. Because of Theorem 7, $n\left(\mu_{n}\{1\}-a_{n}\{1\}\right)$ converges a.s. whenever the distribution of $\mu\{1\}$ is absolutely continuous with an almost Lipschitz density $f$. Our last result, inspired by [22], provides a sharp estimate of $\rho\left(\pi_{n}, \pi_{n}^{*}\right)$ under the assumption that $f$ is Lipschitz (and not only almost Lipschitz).

Theorem 10. Suppose $S=\{0,1\}, X$ exchangeable, and the distribution of $\mu\{1\}$ absolutely continuous with a Lipschitz density $f$. Then,

$$
\rho\left(\pi_{n}, \pi_{n}^{*}\right) \leq \frac{1}{n}\left(1+\frac{c}{3}\right)
$$

for all $n \geq 1$, where $c$ is the Lipschitz constant of $f$.

Proof. Let $\bar{X}_{n}=(1 / n) \sum_{i=1}^{n} X_{i}$ and $V=\lim \sup _{n} \bar{X}_{n}$. Since the $X_{n}$ are indicators,

$$
\mu_{n}\{1\}=\bar{X}_{n}, \quad \mu\{1\}=V \quad \text { and } \quad a_{n}\{1\}=E\left(V \mid \mathcal{G}_{n}\right) \quad \text { a.s. }
$$

Take $Q$ to be the Ferguson-Dirichlet law such that

$$
b_{n}\{1\}=E_{Q}\left(V \mid \mathcal{G}_{n}\right)=\frac{1+n \bar{X}_{n}}{n+2}, \quad Q \text {-a.s. }
$$

Then, $\left|\bar{X}_{n}-E_{Q}\left(V \mid \mathcal{G}_{n}\right)\right| \leq 1 /(n+2)$. Further, since $V$ is uniformly distributed on $[0,1]$ under $Q$,

$$
\begin{aligned}
P\left(X_{1}=x_{1}, \ldots, X_{n}=x_{n}\right) & =\int_{0}^{1} \theta^{k}(1-\theta)^{n-k} f(\theta) d \theta \\
=\int V^{k}(1-V)^{n-k} f(V) d Q= & \int_{\left\{X_{1}=x_{1}, \ldots, X_{n}=x_{n}\right\}} f(V) d Q
\end{aligned}
$$

for all $n \geq 1$ and $x_{1}, \ldots, x_{n} \in\{0,1\}$, where $k=\sum_{i=1}^{n} x_{i}$. Hence, $f(V)$ is a density of $P$ with respect to $Q$. In particular,

Note also that

$$
E\left(V \mid \mathcal{G}_{n}\right)=\frac{E_{Q}\left\{V f(V) \mid \mathcal{G}_{n}\right\}}{E_{Q}\left\{f(V) \mid \mathcal{G}_{n}\right\}} \quad \text { a.s. }
$$

$$
E_{Q}\left\{\left(\bar{X}_{n}-V\right)^{2}\right\}=E_{Q}\left\{E_{Q}\left\{\left(\bar{X}_{n}-V\right)^{2} \mid V\right\}\right\}=E_{Q}\left\{\frac{V(1-V)}{n}\right\}=\frac{1}{6 n} .
$$

Next, define $U_{n}=f(V)-E_{Q}\left\{f(V) \mid \mathcal{G}_{n}\right\}$. Then,

$$
E_{Q}\left\{\bar{X}_{n} U_{n} \mid \mathcal{G}_{n}\right\}=\bar{X}_{n} E_{Q}\left(U_{n} \mid \mathcal{G}_{n}\right)=0, \quad Q \text {-a.s. }
$$


Since $P \ll Q$, then $E_{Q}\left\{\bar{X}_{n} U_{n} \mid \mathcal{G}_{n}\right\}=0$ a.s. with respect to $P$ as well. Hence,

$$
\begin{gathered}
\left|\bar{X}_{n}-E\left(V \mid \mathcal{G}_{n}\right)\right| \leq\left|\bar{X}_{n}-E_{Q}\left(V \mid \mathcal{G}_{n}\right)\right|+\left|E_{Q}\left(V \mid \mathcal{G}_{n}\right)-E\left(V \mid \mathcal{G}_{n}\right)\right| \\
\leq \frac{1}{n+2}+\left|E_{Q}\left(V \mid \mathcal{G}_{n}\right)-\frac{E_{Q}\left\{V f(V) \mid \mathcal{G}_{n}\right\}}{E_{Q}\left\{f(V) \mid \mathcal{G}_{n}\right\}}\right| \\
=\frac{1}{n+2}+\frac{\left|E_{Q}\left\{V U_{n} \mid \mathcal{G}_{n}\right\}\right|}{E_{Q}\left\{f(V) \mid \mathcal{G}_{n}\right\}} \\
=\frac{1}{n+2}+\frac{\left|E_{Q}\left\{\left(V-\bar{X}_{n}\right) U_{n} \mid \mathcal{G}_{n}\right\}\right|}{E_{Q}\left\{f(V) \mid \mathcal{G}_{n}\right\}} \\
\leq \frac{1}{n+2}+\frac{E_{Q}\left\{\left|\left(V-\bar{X}_{n}\right) U_{n}\right| \mid \mathcal{G}_{n}\right\}}{E_{Q}\left\{f(V) \mid \mathcal{G}_{n}\right\}} \text { a.s. }
\end{gathered}
$$

Since $f$ is Lipschitz, one also obtains

$$
\begin{gathered}
E_{Q}\left(U_{n}^{2}\right)=E_{Q}\left\{\left(f(V)-f\left(\bar{X}_{n}\right)-E_{Q}\left\{f(V)-f\left(\bar{X}_{n}\right) \mid \mathcal{G}_{n}\right\}\right)^{2}\right\} \\
\leq 4 E_{Q}\left\{\left(f(V)-f\left(\bar{X}_{n}\right)\right)^{2}\right\} \leq 4 c^{2} E_{Q}\left\{\left(\bar{X}_{n}-V\right)^{2}\right\} .
\end{gathered}
$$

We are finally in a position to estimate $\rho\left(\pi_{n}, \pi_{n}^{*}\right)$. In fact, if $\phi$ is a function on $[0,1]$, with $\phi 1$-Lipschitz and $-1 \leq \phi \leq 1$, then

$$
\begin{gathered}
\left|\pi_{n}(\phi)-\pi_{n}^{*}(\phi)\right|=\left|E\left\{\phi\left(\bar{X}_{n}\right)\right\}-E\left\{\phi\left(E\left(V \mid \mathcal{G}_{n}\right)\right)\right\}\right| \leq E\left|\bar{X}_{n}-E\left(V \mid \mathcal{G}_{n}\right)\right| \\
\leq \frac{1}{n+2}+E\left\{\frac{E_{Q}\left\{\left|\left(\bar{X}_{n}-V\right) U_{n}\right| \mid \mathcal{G}_{n}\right\}}{E_{Q}\left\{f(V) \mid \mathcal{G}_{n}\right\}}\right\} \\
=\frac{1}{n+2}+E_{Q}\left\{f(V) \frac{E_{Q}\left\{\left|\left(\bar{X}_{n}-V\right) U_{n}\right| \mid \mathcal{G}_{n}\right\}}{E_{Q}\left\{f(V) \mid \mathcal{G}_{n}\right\}}\right\} \\
=\frac{1}{n+2}+E_{Q}\left|\left(\bar{X}_{n}-V\right) U_{n}\right| \\
\leq \frac{1}{n+2}+\sqrt{E_{Q}\left\{\left(\bar{X}_{n}-V\right)^{2}\right\} E_{Q}\left(U_{n}^{2}\right)} \\
\leq \frac{1}{n+2}+2 c E_{Q}\left\{\left(\bar{X}_{n}-V\right)^{2}\right\} \\
=\frac{1}{n+2}+\frac{c}{3 n}<\frac{1}{n}\left(1+\frac{c}{3}\right) .
\end{gathered}
$$

The rate provided by Theorem 10 can not be improved. Take in fact $\phi(x)=x^{2} / 2$ and suppose $P$ a Ferguson-Dirichlet law with $a_{n}\{1\}=\frac{1+n \mu_{n}\{1\}}{n+2}$ a.s. Then, since $\mu\{1\}$ is uniformly distributed on $[0,1]$, one obtains

$$
\begin{gathered}
2(n+2) \rho\left(\pi_{n}, \pi_{n}^{*}\right) \geq 2(n+2)\left|\pi_{n}(\phi)-\pi_{n}^{*}(\phi)\right| \\
=(n+2)\left\{E\left(\mu_{n}\{1\}^{2}\right)-E\left(a_{n}\{1\}^{2}\right)\right\} \\
=(n+2) E\left(\mu_{n}\{1\}^{2}\right)-\frac{1+n^{2} E\left(\mu_{n}\{1\}^{2}\right)+2 n E\left(\mu_{n}\{1\}\right)}{n+2} \\
=\frac{4(n+1) E\left(\mu_{n}\{1\}^{2}\right)-2 n E\left(\mu_{n}\{1\}\right)-1}{n+2} \longrightarrow 4 E\left(\mu\{1\}^{2}\right)-2 E(\mu\{1\})=\frac{1}{3} .
\end{gathered}
$$




\section{REFERENCES}

[1] Aldous D.J. (1985) Exchangeability and related topics, Ecole de Probabilites de Saint-Flour XIII, Lect. Notes in Math. 1117, Springer.

[2] Berti P., Rigo P. (1997) A Glivenko-Cantelli theorem for exchangeable random variables, Statist. Probab. Letters, 32, 385-391.

[3] Berti P., Mattei A., Rigo P. (2002) Uniform convergence of empirical and predictive measures, Atti Sem. Mat. Fis. Univ. Modena, 50, 465-477.

[4] Berti P., Pratelli L., Rigo P. (2004) Limit theorems for a class of identically distributed random variables, Ann. Probab., 32, 2029-2052.

[5] Berti P., Crimaldi I., Pratelli L., Rigo P. (2009) Rate of convergence of predictive distributions for dependent data, Bernoulli, 15, 1351-1367.

[6] Berti P., Pratelli L., Rigo P. (2012) Limit theorems for empirical processes based on dependent data, Electron. J. Probab., 17, 1-18.

[7] Berti P., Pratelli L., Rigo P. (2013) Exchangeable sequences driven by an absolutely continuous random measure, Ann. Probab., 41, 2090-2102.

[8] Blackwell D., Dubins L.E. (1962) Merging of opinions with increasing information, Ann. Math. Statist., 33, 882-886.

[9] Cifarelli D.M., Regazzini E. (1996) De Finetti's contribution to probability and statistics, Statist. Science, 11, 253-282.

[10] Cifarelli D.M., Dolera E., Regazzini E. (2016) Frequentistic approximations to Bayesian prevision of exchangeable random elements, Intern. J. Approximate Reasoning, 78, 138-152.

[11] Crane H. (2016) The ubiquitous Ewens sampling formula, Statist. Science, 31, 1-19.

[12] De Blasi P., Favaro S., Lijoi A., Mena R.H., Prunster I., Ruggiero M. (2015) Are Gibbs-type priors the most natural generalization of the Dirichlet process ?, IEEE Trans. Pattern Anal. Machine Intell., 37, 212-229.

[13] Diaconis P., Freedman D. (1986) On the consistency of Bayes estimates, Ann. Statist., 14, $1-26$.

[14] Dudley R.M. (1999) Uniform central limit theorems, Cambridge University Press.

[15] Efron B. (2003) Robbins, empirical Bayes and microarrays, Ann. Statist., 31, 366-378.

[16] Fortini S., Ladelli L., Regazzini E. (2000) Exchangeability, predictive distributions and parametric models, Sankhya A, 62, 86-109.

[17] Gaenssler P., Stute W. (1979) Empirical processes: a survey of results for independent and identically distributed random variables, Ann. Probab., 7, 193-243.

[18] Ghosal S., van der Vaart A. (2017) Fundamentals of nonparametric Bayesian inference, Cambridge University Press.

[19] Hjort N.L., Holmes C., Muller P., Walker S.G. (2010) Bayesian nonparametrics, Cambridge University Press.

[20] Kingman J.F.C. (1975) Random discrete distributions (with discussion), J. Royal Stat. Soc. $B, 37,1-22$.

[21] Kuelbs J., Dudley R.M. (1980) Log log laws for empirical measures, Ann. Probab., 8, 405-418.

[22] Mijoule G., Peccati G., Swan Y. (2016) On the rate of convergence in de Finetti's representation theorem, ALEA (Lat. Am. J. Probab. Math. Stat.), 13, 1165-1187.

[23] Phadia E.G. (2016) Prior processes and their applications (Second Edition), Springer.

[24] Pitman J., Yor M. (1997) The two-parameter Poisson-Dirichlet distribution derived from a stable subordinator, Ann. Probab., 25, 855-900.

[25] Robbins H. (1964) The empirical Bayes approach to statistical decision problems, Ann. Math. Statist., 35, 1-20.

[26] Sethuraman J. (1994) A constructive definition of Dirichlet priors, Stat. Sinica, 4, 639-650.

[27] van der Vaart A., Wellner J.A. (1996) Weak convergence and empirical processes, Springer.

[28] Zabell S.L. (2005) Symmetry and its discontents (Essays on the history of inductive probability), Cambridge University Press. 
Patrizia Berti, Dipartimento di Matematica Pura ed Applicata "G. Vitali", UniverSita' di Modena e Reggio-Emilia, via Campi 213/B, 41100 Modena, Italy

E-mail address: patrizia.berti@unimore.it

Luca Pratelli, Accademia Navale, viale Italia 72, 57100 Livorno, Italy

E-mail address: pratel@mail.dm.unipi.it

Pietro Rigo (corresponding author), Dipartimento di Matematica "F. Casorati", Universita' di Pavia, via Ferrata 1, 27100 Pavia, Italy

E-mail address: pietro.rigo@unipv.it 